\title{
Sistema Embebido para Validar el Funcionamiento de la Tarjeta de Adquisición de Datos USB-6009 de National Instruments
}

\author{
Francisco J. Enríquez, Ernesto Sifuentes, Gabriel Bravo y Arnulfo Castro \\ Universidad Autónoma de Ciudad Juárez, Instituto de Ingeniería y Tecnología \\ Departamento de Ingeniería Eléctrica y Computación, Ciudad Juárez, Chihuahua, México \\ (e-mail: fenrique@uacj.mx, esifuent@uacj.mx,gbravo@uacj.mx y arncastr@uacj.mx)
}

Recibido Dic. 7, 2015; Aceptado Feb. 11, 2016; Versión final Abr. 10, 2016, Publicado Oct. 2016

\begin{abstract}
Resumen
Se construyó un sistema de prueba semi-automatizado para auxiliar en las labores de préstamos de equipo del Laboratorio de Control en la Universidad Autónoma de Ciudad Juárez (México), específicamente de las tarjetas de adquisición de datos USB-6009 del fabricante National Instruments. Se diseñó un sistema para validar las entradas y salidas de esta tarjeta. El desarrollo se llevó a cabo utilizando una interfaz gráfica en LabVIEW y un punto de apoyo o base prototipo basada en un microcontrolador, que ayuda a corroborar su correcto o mal funcionamiento de la tarjeta cuando es prestada o cuando es retornada por el usuario al laboratorio. El diseño de la interfaz indica al usuario los periféricos que estén dañados y genera un reporte para llevar el control de las validaciones realizadas. De esta manera se tendrá un mejor control de préstamo de estas tarjetas de adquisición al momento de su entrega y devolución, beneficiando al laboratorio y al usuario.
\end{abstract}

Palabras clave: tarjeta de adquisición de datos; USB-6009; LabVIEW; microcontrolador; automatización

\section{Embedded System to Validate the Operation of the Data Acquisition Card USB-6009 of National Instrument}

\begin{abstract}
A semi-automated test system to assist on the task of controlling the loans of equipment and devices in the Control Laboratory at the Autonomous University of Ciudad Juarez (Mexico), specifically of the data acquisition card USB-6009 of National Instrument, was constructed. A system to validate the inputs and outputs of the card from the Lab. was designed. The development was done using a graphical interface in LabVIEW and a mount prototype based in a microcontroller, to help in checking the correct or incorrect functioning of the card when it is borrowed and when is returned by the user to the Lab. The interface will inform the user of those peripherals that are damaged and generate a report to keep track of the validations performed. In this way better control of the lending process of these boards is achieved, with benefits for both the Lab and the users.
\end{abstract}

Keywords: data acquisition card; USB-6009; LabVIEW; microcontroller; automation 


\section{INTRODUCCIÓN}

Las tarjetas de adquisición de datos son dispositivos diseñados para la adquisición y medición de señales que consiste en la toma de muestras de variables físicas del mundo real (sistema analógico por lo general), para generar datos que pueden ser manipulados por un programa diseñado en un ordenador. El dispositivo de adquisición de datos o USB-6009 es un dispositivo de bajo costo ideal para realizar mediciones de entradas analógicas (ocho canales) y con funcionalidad para generar niveles de voltaje de salida (dos canales), dispone de un contador y dos puertos con 8 líneas de entradas y salidas digitales. Esta funcionalidad se puede realizar simultáneamente para solucionar aplicaciones que van desde registros de datos, adquisición analógica con alarmas, conteo de eventos, hasta control de lazo cerrado. Al usar la interfaz de programación LabVIEW se puede lograr desarrollar este tipo de aplicaciones de manera más sencilla y además permite la flexibilidad de expandir la funcionalidad (Instruments, 2013).

Existen diferentes tipos de automatización para validación, esto depende de las herramientas que necesitemos utilizar, por ejemplo, está el trabajo "Dispositivo para la Evaluación y Diagnóstico del Estado del cabezal en Centros de Mecanizado de Alta Velocidad", en el cual se presenta un dispositivo portátil, capaz de identificar el estado del cabezal en máquinas-herramienta de alta velocidad, al finalizar una prueba, la aplicación es capaz de mostrar la señal de vibración RMS y evaluarla según los límites establecidos. El sistema fue práctico, intuitivo y completamente independiente de la máquina, capaz de realizar un diagnóstico fiable a partir de una máquina en funcionamiento (Ramírez, et al, 2006).

También existe el desarrollo de una aplicación y validación de una metodología para la evaluación de la usabilidad de interfaces de sistemas de supervisión industrial, donde se exponen los aspectos fundamentales de evaluación de usabilidad de sistemas basados en software. La metodología fue aplicada en un laboratorio a varias interfaces de supervisión de un proceso de dosificación de líquidos. La metodología desarrollada, hace parte de las técnicas automatizadas de acuerdo con la clasificación que se presenta en el artículo y además incorpora a los usuarios (operadores) para extraer información durante la interacción en tiempo de ejecución en actividades de supervisión de procesos lo cual fue una innovación (Hurtado, et al, 2013).

En el trabajo de (Yao, et al, 2015) se diseñó un sistema basado en computadora para pruebas automatizadas a válvulas de combustible en aviación, el cual prueba su eficiencia y exactitud, esto con la finalidad de detectar características no lineales que afecten el rendimiento del uso de las mismas. El sistema consiste principalmente en una computadora industrial, una tarjeta de adquisición de datos, un circuito acondicionador de señales y el software de pruebas desarrollado en LabVIEW. Comparado con la instrumentación tradicional, este sistema tiene una estructura flexible, por lo cual es fácil de integrar y expandir, esto se logra al utilizar un diseño de hardware modular. Después de varias pruebas se encontró la relación entre el circuito de la bobina de la válvula y el circuito de control eléctrico, lo cual fue clave para obtener los efectos ideales de la prueba de rendimiento.

Para caracterizar el rendimiento de un transmisor-receptor óptico de manera rápida y con una alta exactitud y confiabilidad, fue necesario diseñar un sistema de prueba basado en LabVIEW por parte de investigadores del National Key Laboratory on High Power Semiconductor. El sistema prueba la potencia óptica, el rango de extinción y el tiempo de subida y bajada de la señal emitida por el transmisor. Fueron probados módulos de transmisión óptica paralela de alta velocidad y el resultado de las pruebas mostraron el rango de extinción aproximado de $8.5-10 \mathrm{~dB}$, la potencia de cada canal de alrededor de $0.87-0.977 \mathrm{~mW}$ y que las velocidades de transmisión de datos pueden alcanzar valores de hasta $30 \mathrm{Gbps}$. El sistema pudo simplificar el proceso de prueba de los módulos e incrementar la eficiencia y exactitud de las pruebas (Yu, et al, 2015).

(Jinmei, 2015) presenta el proceso de implementación de un sistema remoto controlador del bus 1553B. LabVIEW fue adoptado para realizar la función en el controlador principal y probador de terminales en sistemas de prueba a equipo electrónico. El sistema es capaz de almacenar las pruebas de los datos así como el estado de la comunicación de los mismos en tiempo real, por lo que este sistema de prueba de equipo electrónico es conveniente para el monitoreo de datos. El resultado de las pruebas arrojó que el sistema trabajó de manera eficiente con una baja tasa de error de bit. Debido a que el trabajo se realizó con una metodología estandarizada de diseño modular y concluye que el sistema es capaz de expandirse a otras aplicaciones.

En instituciones académicas se desarrollan sistemas semi-automatizados que son implementados para obtener datos escolares de una manera más eficientes, o de probar material o dispositivos electrónicos en los laboratorios al momento de prestarlos a los alumnos como está el caso del sistema de pruebas para la validación de las tarjetas de adquisición de datos USB-6009 que utiliza el laboratorio de control en IIT-UACJ. 
Este sistema funciona con dos USB-6009, una funciona como dispositivo patrón y la otra tarjeta es la que se sometería a prueba. Utiliza una interfaz de usuario programada en LabVIEW que le indica al usuario los periféricos dañados de la tarjeta y genera un reporte cada vez que se realiza una validación a las tarjetas (Gurrola et al., 2013).

\section{DIAGRAMA A BLOQUES DEL SISTEMA}

El dispositivo de adquisición de datos o USB-6009 es un dispositivo de bajo costo ideal para crear mediciones de entradas analógicas y con funcionalidad para generar niveles de voltaje de salida, dispone de un contador y dos puertos con 8 líneas de entradas y salidas digitales. Esta funcionalidad se puede realizar simultáneamente para solucionar aplicaciones que van desde registros de datos, adquisición analógica con alarmas, conteo de eventos, hasta control de lazo cerrado (Intruments, 2013).

Al usar la interfaz de programación LabVIEW se puede lograr desarrollar este tipo de aplicaciones de manera más sencilla y además permite la flexibilidad de expandir la funcionalidad. Los periféricos de este dispositivo están divididos en cuatro partes: puertos digitales, puertos de entradas analógicas, puerto de salidas analógicas, y el contador. El dispositivo es alimentado directamente desde un puerto USB de la computadora haciéndolo más portátil y está diseñado para el ámbito estudiantil y para proyectos pequeños que no requieren de muy alta velocidad de adquisición. La tarjeta de adquisición de datos USB-6009 dispone de 8 entradas analógicas y 2 salidas analógicas, 12 puertos digitales, 1 contador de 32 bits y 2 salidas de voltaje de $+5 \mathrm{~V}$ y $+2.5 \mathrm{~V}$ (Instruments, 2013). En la figura 1 se puede apreciar la imagen de una tarjeta USB-6009. Para realizar las pruebas se transmitirán y recibirán datos entre los diferentes puertos de la tarjeta de prueba y un microcontrolador $(\mu \mathrm{C})$, el cual ayudará a realizar el proceso de lectura y escritura de todos los puertos de la tarjeta USB-6009 controlado a través de una interfaz de usuario previamente programada en LabVIEW que se encargará de determinar cuáles puertos funcionan correctamente y cuáles no.

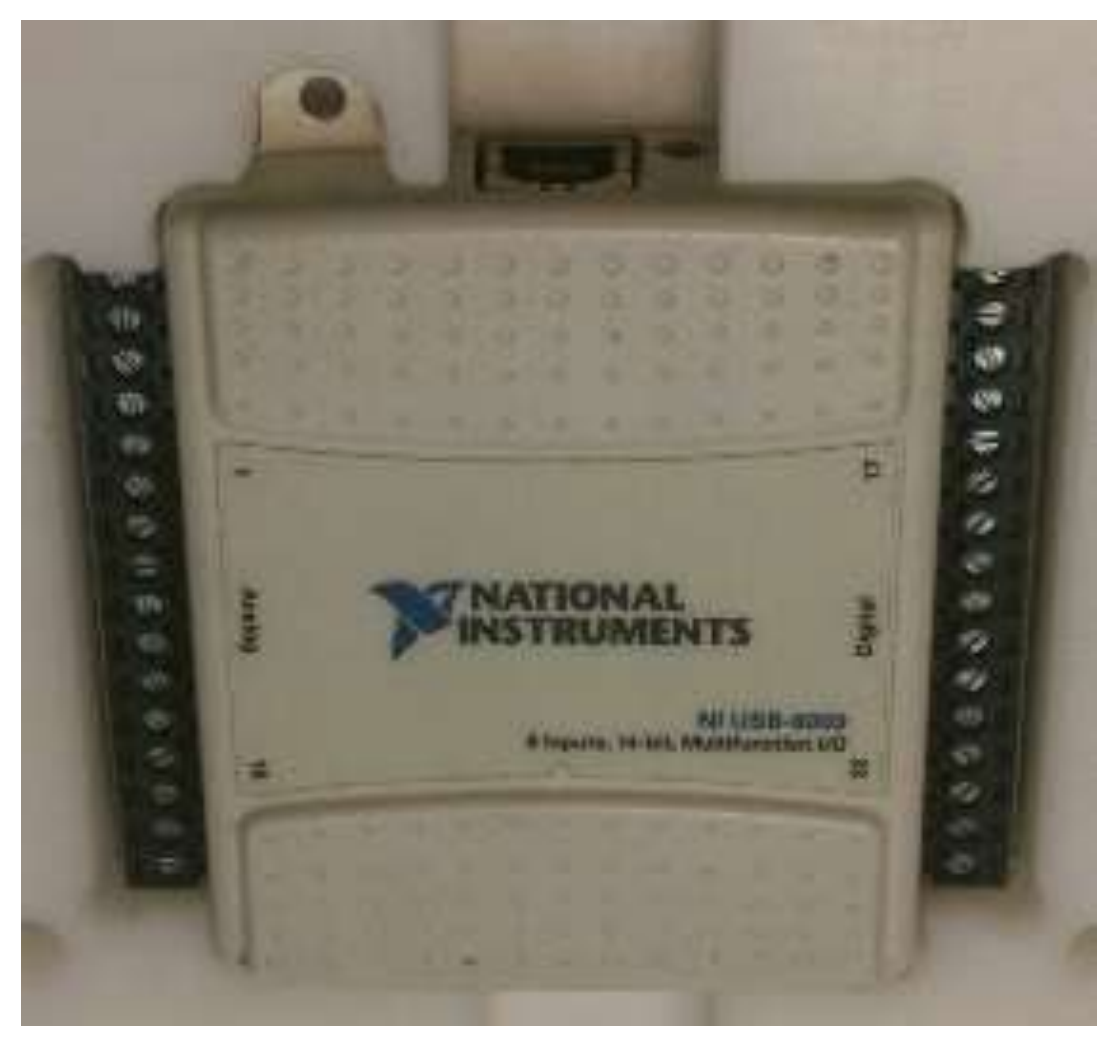

Fig. 1: Tarjeta USB-6009.

El objetivo de diseñar la interfaz de usuario en LabVIEW, es facilitar la implementación de la misma y el manejo de la tarjeta de prueba. La interfaz de usuario debe de ser amigable y fácil de manejar por cualquier persona con conocimiento básico sobre el sistema de prueba. La figura 2 muestra de manera general el método de conexión entre los dispositivos del sistema de prueba, en ella se puede apreciar que debe existir una comunicación entre la tarjeta USB-6009 y el sistema presentado en este trabajo. 


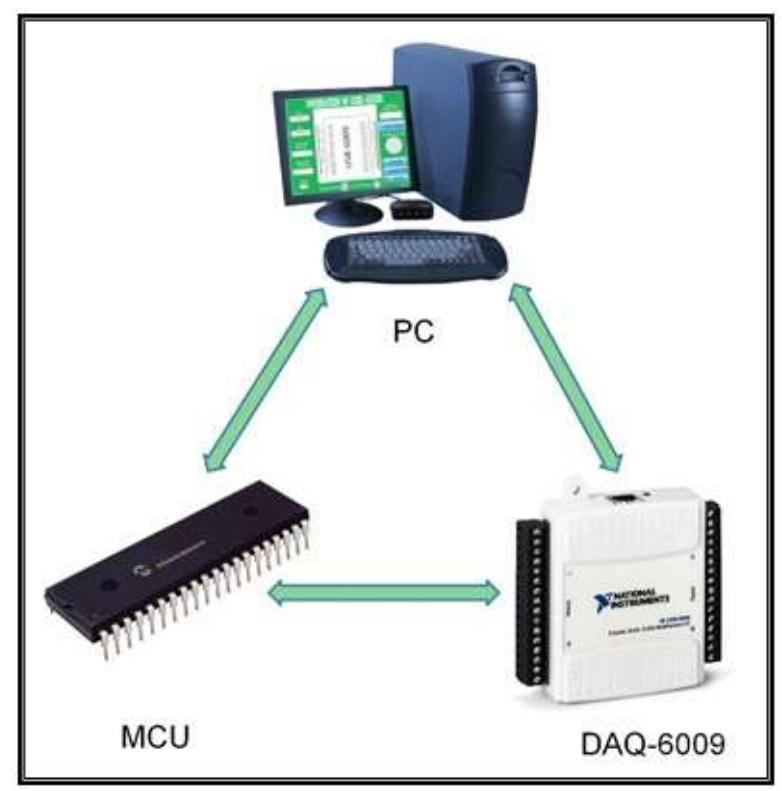

Fig. 2: Diagrama general de conexión.

\section{PRUEBAS AL DISPOSITIVO}

Como la DAQ NI USB-6009 consta de diferentes puertos se tendrán que realizar pruebas específicas para cada uno de ellos. Para realizar la prueba de los puertos digitales, el $\mu \mathrm{C}$ envía señales lógicas a través de sus puertos digitales para que sean leídas por las entradas digitales de la DAC USB-6009, después la tarjeta de adquisición hará la misma tarea enviando datos al $\mu \mathrm{C}$ (ambos dispositivos realizarán la función de leer y escribir), de esta manera se habrá logrado probar las funciones que realizan los puertos digitales de la tarjeta de prueba.

Para realizar la prueba de las salidas analógicas, la aplicación de prueba enviará voltajes específicos que serán leídos a través de las entradas analógicas del $\mu \mathrm{C}$, del mismo modo se probaran las señales de $+2.5 \mathrm{~V}$ $y+5 \mathrm{~V}$. En el caso de la prueba de las entradas analógicas, se hará un proceso inverso al que se realizó al probar las salidas analógicas. El $\mu \mathrm{C}$ será el encargado de mandar ciertos voltajes específicos para que la tarjeta de prueba los lea, si el voltaje leído es el correcto, se indicara que la prueba es correcta, para esto se da un margen de error en las lecturas de medio volt ya que al tener diferentes resoluciones entre el sistema a probar y el diseñado existe una discrepancia entre el valor de la señal escrita y la leída.

Para probar el contador de la tarjeta de prueba, el $\mu \mathrm{C}$ enviará un tren de pulsos por un pin digital, el cual hará que el contador se incremente un número finito de veces. El $\mu \mathrm{C}$ seleccionado para la implementación del prototipo fue el PIC18F4550 por las siguientes razones: (i) Cumple la mayoría de los requerimientos que se necesitan; (ii) El programador es fácil de conseguir y económico; (iii) Es un $\mu \mathrm{C}$ común, existe mucha información y ejemplos sobre cómo programar los PIC18 en general; (iv) El entorno de desarrollo integrado (IDE), es software libre y se puede descargar gratis de su página oficial; (v) La versión estudiantil del compilador se puede descargar gratis de su página oficial; (vi) El $\mu \mathrm{C}$ cuenta con una función llamada "autoescritura" lo que permite actualizar el firmware utilizando un bootloader sin la necesidad del programador; y (vii) Cuenta con la cantidad adecuada de entradas/salidas digitales y entradas analógicas.

Ya que el $\mu \mathrm{C}$ no dispone de un convertidor D/A para realizar la prueba de las entradas analógicas se decidió utilizar el TLV5628 ya que cumple con las características necesarias, como son: 8 canales, funciona con alimentación unipolar de 3V, dispone de buffer en la salida y la interfaz de comunicación es SPI, por lo que solo se requieren 3 pines (Instrument, 1994). Para la comunicación con la PC se decidió utilizar el convertidor USB a RS-232 con circuito integrado CP2102, ya que es de bajo costo, pequeño y dispone de los controladores para Windows XP hasta el W8 (Laboratories, 2013).

\section{DISEÑO DEL CIRCUITO IMPRESO}

Se utilizó el software Eagle para diseñar el circuito impreso (PCB), partiendo del diagrama que se realizó en ese mismo programa, además se añadieron las iniciales de la universidad en la capa inferior, como se muestra en la figura 3 (Eagle, 2011). 


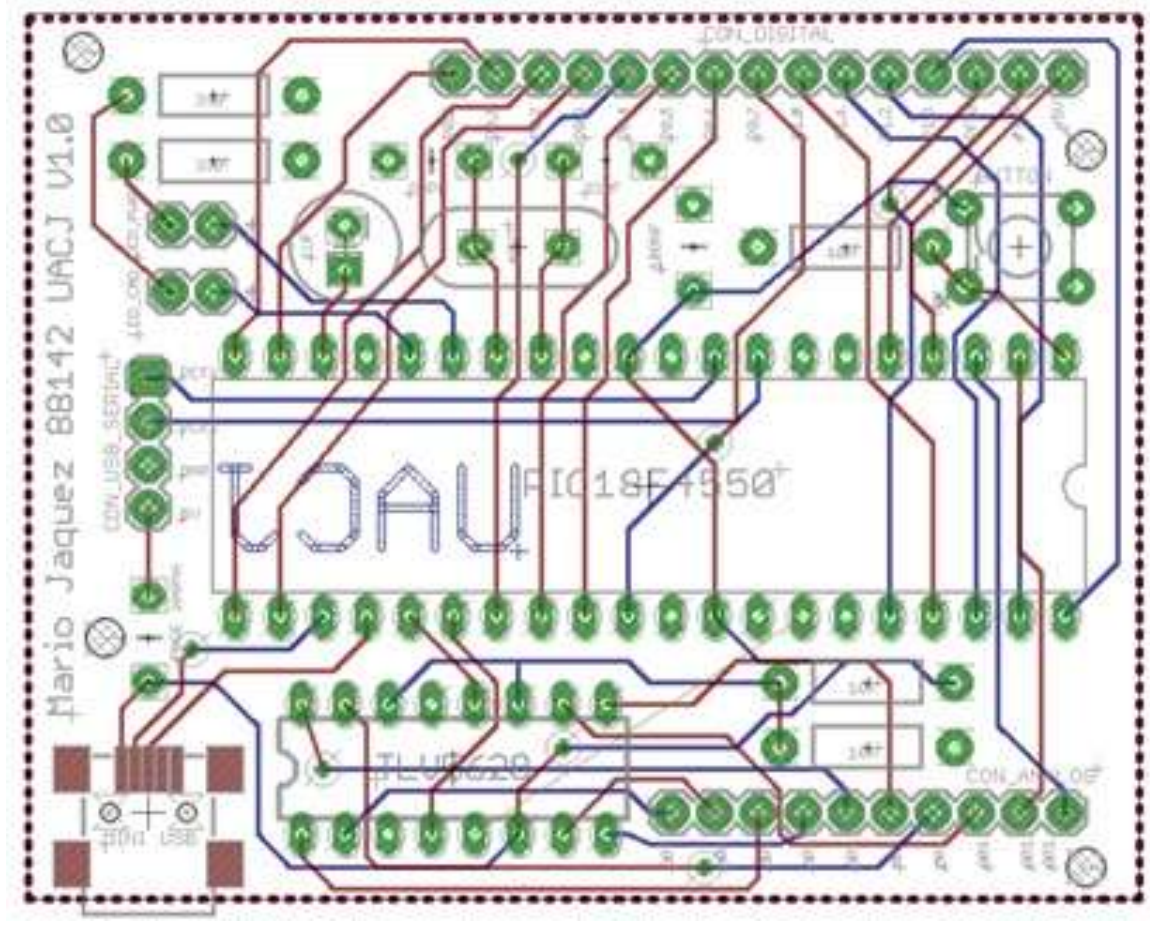

Fig. 3: Componentes organizados y ruteados.

La placa se mandó a fabricar a una comunidad dedicada a la fabricación de pequeños prototipos llamada "Osh Park". El costo es de $\$ 5.00$ dlls. por pulgada cuadrada y se entregan tres copias de la tablilla sin costo adicional en tablillas de dos capa (Park, 2013). Al momento de entregarles el diseño para su fabricación en su página oficial ellos te muestran un preliminar de cómo se vería una vez fabricada, que ayuda a detectar posibles errores antes de que sea enviado, como se muestre en la figura 4.
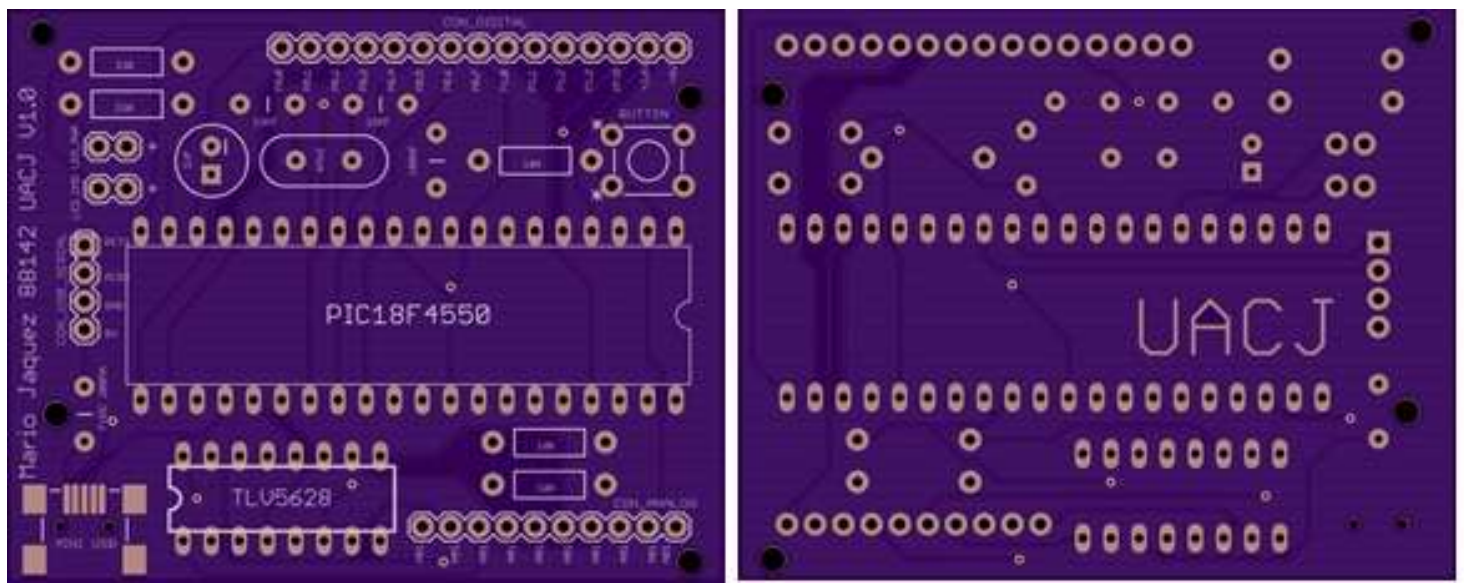

Fig. 4: PCB preliminar del fabricante, capas superior e inferior.

\section{DISEÑO DE LA BASE DEL PROTOTIPO}

La base del prototipo quedará fija para evitar movimientos, será de fácil uso para el usuario y solo se limitará a cambiar la USB-6009 cada vez que se realice una validación. Entonces el prototipo constará de dos partes: superior e inferior.

Superior: En la parte superior se pretende montar toda la circuitería y los cables. Tendrá que tener un tamaño mayor al ancho del PCB diseñado para facilitar su ensamble y el de los cables que van hacia el circuito. También estarán los pernos que harán contacto con los tornillos de la tarjeta de prueba y los pernos guías. Se colocó un perno en cada esquina de la base para que esté fija a la parte inferior, así solo se quitará la parte superior en cada validación. Se hizo una cavidad en donde se acopla el PCB y un orificio donde irá el conector USB-SERIAL. El diseño de la parte superior con sus medidas en pulgadas se muestra en la figura 5 . 


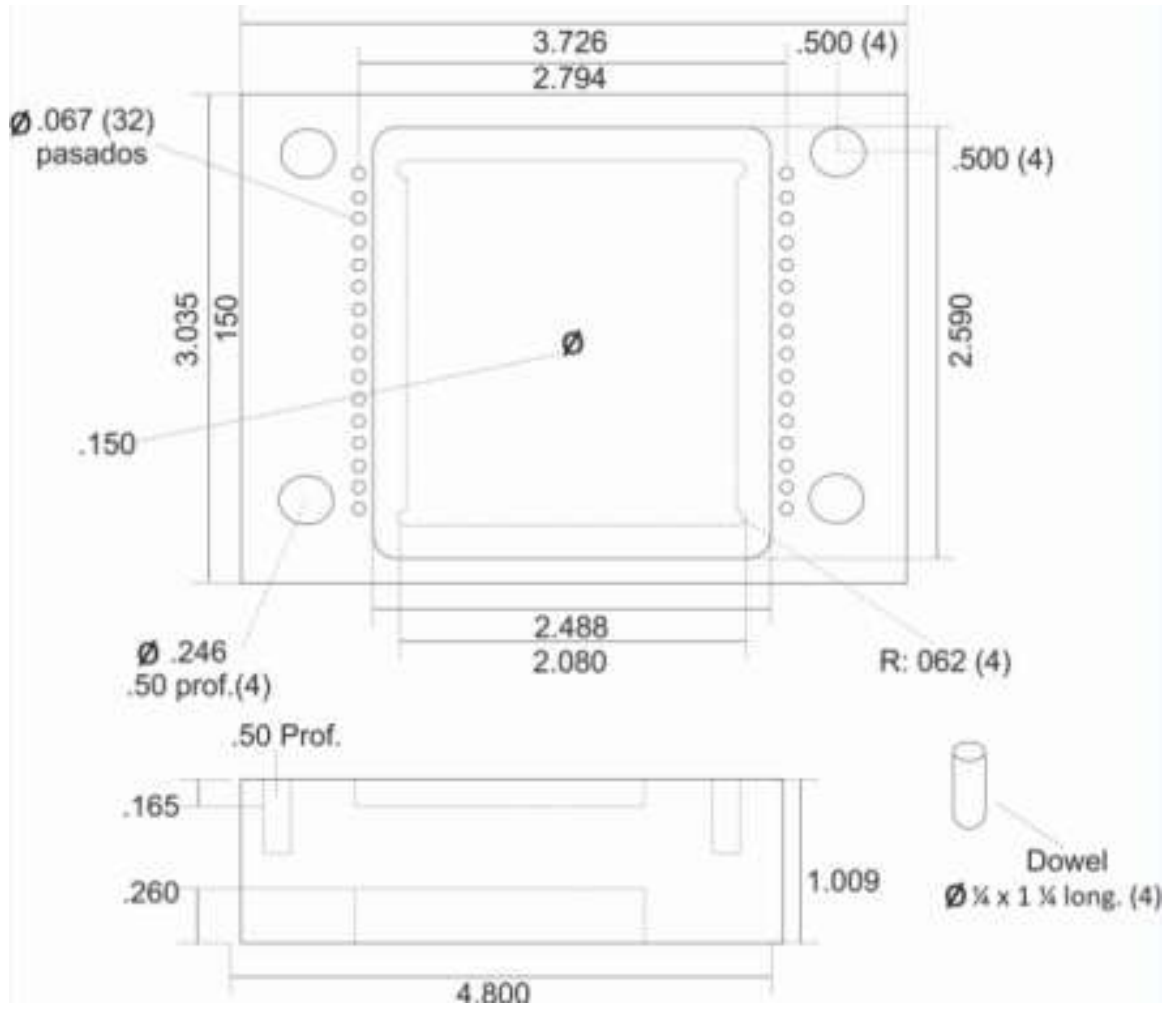

Fig. 5: Diseño de la parte superior de la base, medidas en pulgadas.

Inferior: En la parte inferior será el "Holder" donde se alojara la USB-6009, las medidas del molde serán casi a las medidas del contorno de la tarjeta para que no quede muy holgado y así evitar que se mueva. En esta parte irán los orificios donde acoplaran los pernos guías de cada esquina por lo que deben de ser poco más anchos para que sea fácil a extracción de la parte superior. Se tomaron las medidas de referencia que brinda la hoja de datos del dispositivo para crear el molde donde irá acoplada la USB-6009. En la figura 6 se muestra el diseño de la parte inferior con sus medidas en pulgadas:

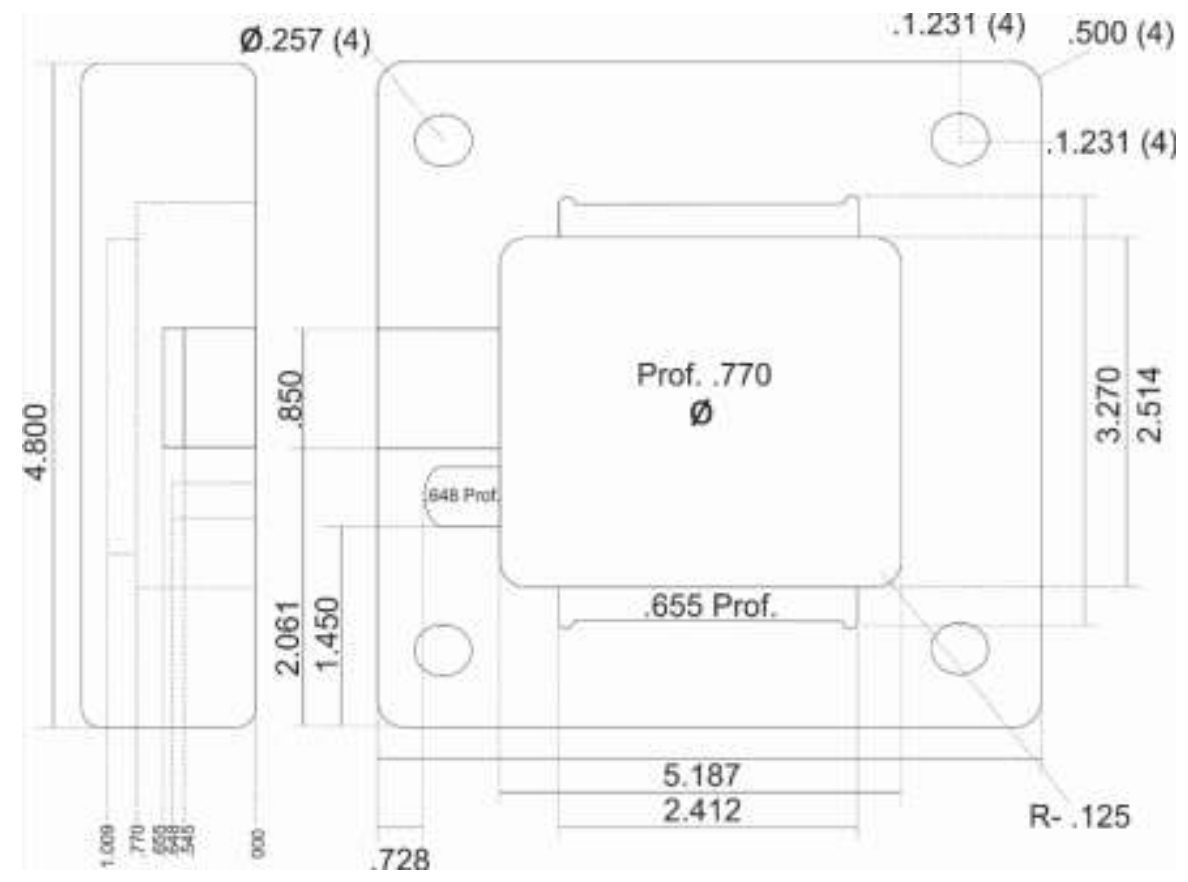

Fig. 6: Diseño de la parte inferior de la base, medidas en pulgadas.

Se envió a fabricar la base a una empresa que se dedica a diseñar ("holders") para conectores de arneses. Se utilizó polioximetileno (Delrin) porque es un material utilizado en la empresa para fabricar piezas de alta precisión con una alta rigidez y baja fricción. Para la fabricación de la parte superior los pernos guías a utilizar son unos cilindros metálicos (Dowel), que irán acoplados en los orificios de 1/4" de diámetro por 11/4"de longitud. Los pernos hacen contacto con los tornillos de la tarjeta de prueba y son pernos retractiles con resorte. 
Estos pernos son utilizados en la empresa para pruebas eléctricas de conectores por lo que son ideales para este prototipo.

\section{DISEÑO DEL FIRMWARE DEL $\mu \mathrm{C}$}

El código del Firmware se implementó en $C$ y se utilizó el entorno de programación MPLAB X IDE y el compilador C18 para el lenguaje de programación C. El diseño del Firmware se divide en tres etapas principales. (i) Configuración inicial y estado predeterminado del sistema: En esta etapa se configuraran periféricos para que operen de acuerdo a las especificaciones requeridas así como poner en estado predeterminado todos los puertos. El estado predeterminado se refiere a que todos los puertos digitales por default son entradas para proteger el $\mu \mathrm{C}$; (ii) Esperando a recibir un comando: En esta sección la aplicación quedará en espera de recibir un comando de los preestablecidos en la tabla 1, si se llega a recibir un comando no válido solamente lo ignorará; y (iii) Ejecutar comando recibido: Una vez recibido un comando válido procederá a ejecutar el bloque de instrucciones correspondiente al comando.

El comando 1 consiste en enviar una cadena de texto que corresponde a la versión del Firmware cargado en el $\mu \mathrm{C}$, con el siguiente formato: "FIRMWARE_v1.0_[MM/DD/AAAA]" que se utilizará para buscar el puerto donde se encuentra conectado el dispositivo. Como el $\mu \mathrm{C}$ ejecutará cada una de las instrucciones 0 comandos que se le darán por el puerto serie, se enumeró cada comando y se definió la función que realizará para ayudar a realizar la prueba correspondiente. La lista de comandos se presenta en la tabla 1.

Tabla 1: Lista de comandos.

\begin{tabular}{|c|c|c|c|}
\hline Hexadecimal & Decimal & Función & Respuesta \\
\hline $0 \times 01$ & 1 & Versión del firmware & Una cadena de texto \\
\hline $0 \times 02$ & 2 & Poner PORTD $<7-0>$ y PORTB $<7-4>$ salidas y escribir unos & - \\
\hline $0 \times 03$ & 3 & Poner PORTD $<7-0>$ y PORTB $<7-4>$ salidas y escribir ceros & - \\
\hline $0 \times 04$ & 4 & Poner PORTD $<7-0>$ y PORTB $<7-4>$ entradas & - \\
\hline $0 \times 05$ & 5 & Leer el bit PORTD $<0>$ & 0,1 \\
\hline $0 \times 06$ & 6 & -eer el bit PORTD $<1>$ & 0,1 \\
\hline $0 \times 07$ & 7 & -eer el bit PORTD<2> & 0,1 \\
\hline $0 \times 08$ & 8 & -eer el bit PORTD $<3>$ & 0,1 \\
\hline $0 \times 09$ & 9 & -eer el bit PORTD<4> & 0,1 \\
\hline $0 \times 0 \mathrm{~A}$ & 10 & -eer el bit PORTD<5> & 0,1 \\
\hline $0 \times 0 \mathrm{~B}$ & 11 & -eer el bit PORTD<6> & 0,1 \\
\hline $0 \times 0 \mathrm{C}$ & 12 & -eer el bit PORTD<7> & 0,1 \\
\hline $0 \times 0 \mathrm{D}$ & 13 & -eer el bit PORTB<4> & 0,1 \\
\hline $0 \times 0 \mathrm{E}$ & 14 & -eer el bit PORTB $<5>$ & 0,1 \\
\hline $0 \times 0 \mathrm{~F}$ & 15 & -eer el bit PORTB<6> & 0,1 \\
\hline $0 \times 10$ & 16 & -eer el bit PORTB $<7>$ & 0,1 \\
\hline $0 \times 11$ & 17 & -eer el voltaje en ANO & Voltaje leído \\
\hline $0 \times 12$ & 18 & Leer el voltaje en AN1 & Voltaje leído \\
\hline $0 \times 13$ & 19 & Leer el voltaje en AN2 & Voltaje leído \\
\hline $0 \times 14$ & 20 & Leer el voltaje en AN3 & Voltaje leído \\
\hline $0 \times 15$ & 21 & Poner voltaje en la salida $\mathrm{X}$ del convertidor D/A & - \\
\hline $0 \times 16$ & 22 & Enviar tren de pulsos & - \\
\hline
\end{tabular}

\section{DISEÑO DE LA APLICACIÓN EN LabVIEW}

Para el diseño de la aplicación se utilizó un diagrama de capas en donde se considera lo siguiente: (i) Los bloques naranjas del manejo del $\mu \mathrm{C}$, se encargan de administrar los comandos que soporta el $\mu \mathrm{C}$ y también la comunicación vía serial; (ii) El manejo de la USB-6009 es para la tarjeta de prueba, esta se segmentó en cuatro bloques: 1) El primero se encarga de leer o escribir en los puertos digitales, 2) la segunda de leer el valor en las entradas analógicas, 3) el tercer bloque escribirá el voltaje en las salidas analógicas y por último 4) el cuarto bloque realizará la función de buscar la USB-6009; (iii) Los bloques de pruebas utilizan las capas anteriores y se encargan de realizar las pruebas de cada uno de los periféricos de la tarjeta de prueba; y (iv) Por último la capa de Interfaz es la que interactuará con el usuario y administra el resto de los bloques para realizar las pruebas. El diagrama de capas de programación en LabVIEW se presenta en la figura 7 y la secuencia de prueba se muestra en la figura 8. 


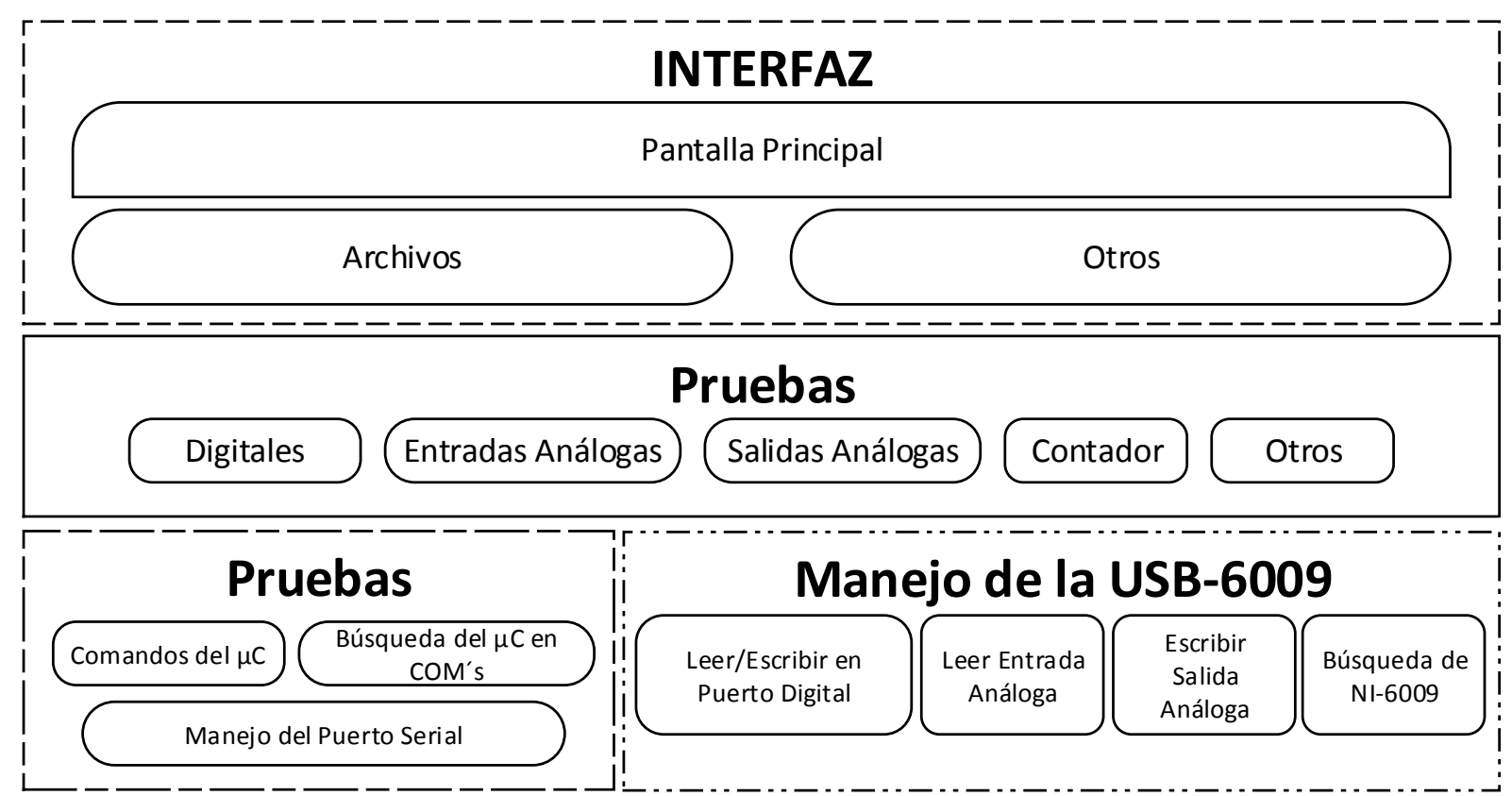

Fig. 7: Capas del diseño de la aplicación en LabVIEW.

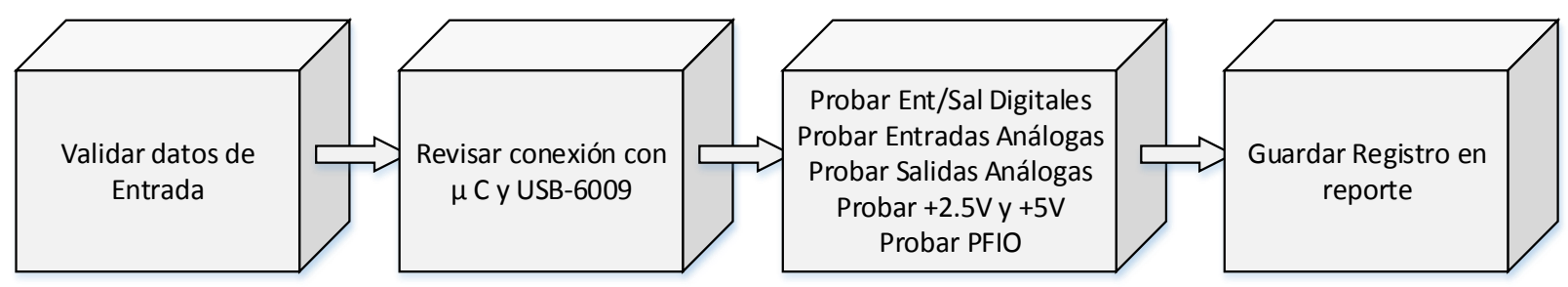

Fig. 8: Diagrama a bloques de la secuencia de prueba.

Se generó un archivo de instalación de la aplicación en LabVIEW 2013, donde se encuentran el archivo ejecutable, los drivers necesarios y el Run-Time Engine el cual es un software necesario para correr programas ejecutables realizados en LabVIEW de la compañía National Instruments, esto para que la aplicación se pueda ejecutar en cualquier computadora aunque no esté instalado el LabVIEW, la aplicación muestra la fecha y hora que se utilizan para el registro de las pruebas que se realicen. Para capturar los datos principales como el nombre de usuario, matrícula y numero de dispositivo se utilizaron cajas de texto los cuales son verificados antes de realizar una prueba asegurándose de que ninguno este en blanco. Se utilizaron cuatro botones para el manejo de la aplicación, uno de ellos se encarga de realizar la prueba, el otro borrar los datos, otro para abrir el reporte de pruebas y por último salir de la aplicación. Se colocaron indicadores en cada uno de los pines de la imagen de la tarjeta de prueba que se probarán, teniendo un color gris como estado inactivo, un color verde si pasa la prueba y rojo si no. Otro indicador principal el cual mostrará el resultado de la validación del dispositivo y dos indicadores más indicando el estado de conexión de la tarjeta de prueba y del $\mu \mathrm{C}$. La interfaz se expone en la figura 9.

\section{RESULTADOS Y DISCUSIÓN}

A diferencia del trabajo presentado por Gurrola et al., en el 2013, este proyecto no necesita del uso de otra tarjeta USB-6009, lo que permite que otro estudiante utilice esa tarjeta que se empleaba, además no se contaba con una base a prueba de errores, ya que solo se intercambiaban los conectores de la tarjeta a probar, pudiendo ocasionar un accidente en caso de una mala instalación de los mismos. En la figura 10 se exhibe el resultado del ensamblaje de la tablilla que suple a la USB-6009. La primer prueba en hardware fue la de montar la base de forma incorrecta para verificar que el perno guía realizara bien su función que es la de indicar al usuario que la base este mal colocada y de también de no dejar que los pines hagan contacto en ningún momento para evitar que el circuito se dañe, como se muestra en la figura 11. La segunda prueba fue la de verificar el sistema con los tornillos que tiene la USB-6009 a diferentes niveles de distancia para saber si en realidad existe contacto y evitar dar un resultado incorrecto al momento de la validación. Esta prueba se realizó utilizando únicamente los puertos digitales. La prueba revelo que es necesario tener los tornillos totalmente cerrados para que exista buena conexión. 


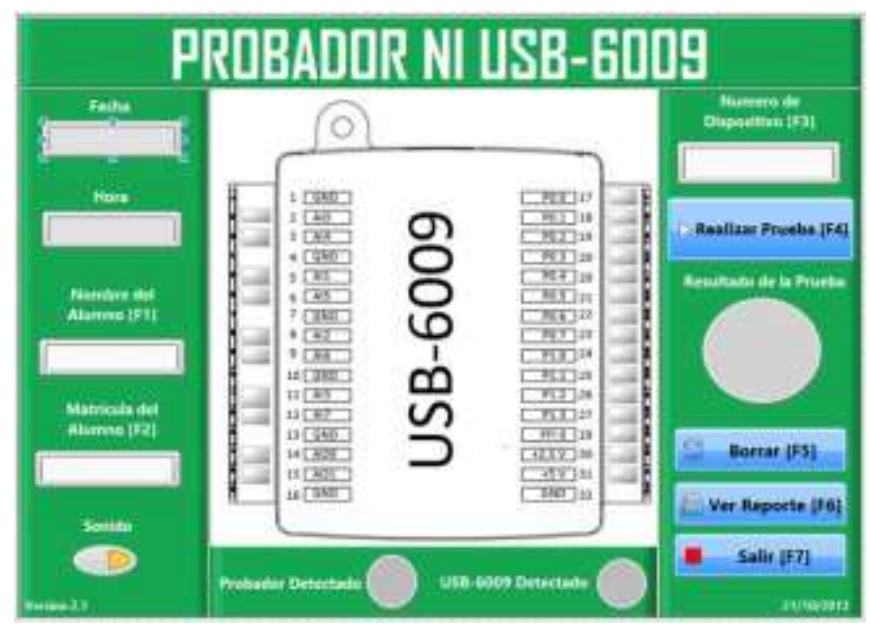

Fig. 9: Interfaz de usuario.

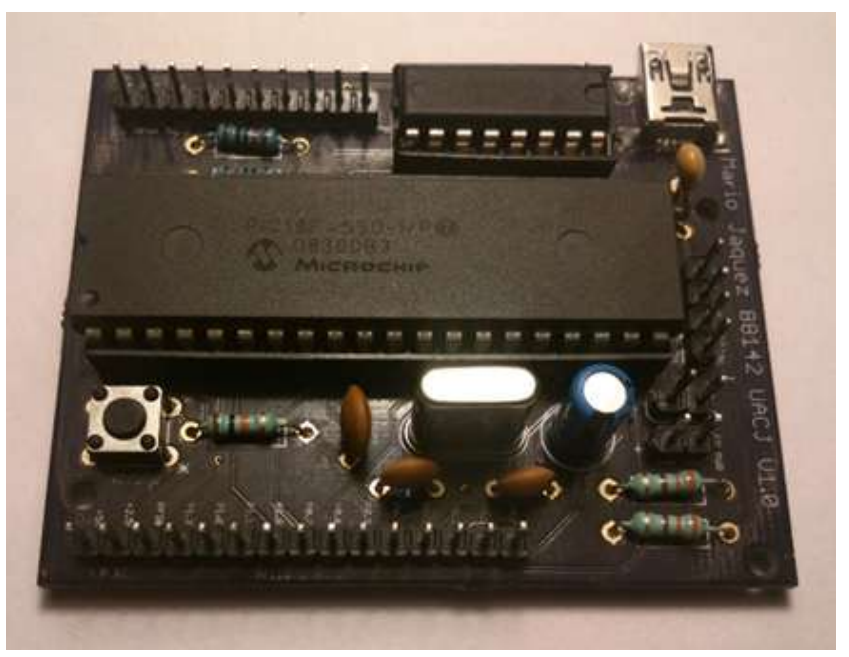

Fig. 10: Tablilla ensamblada con todos los componentes.

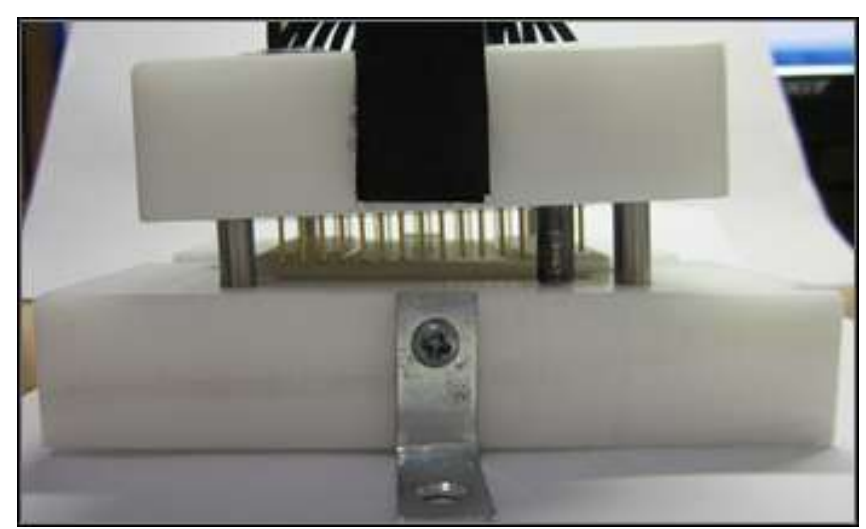

Fig. 11: Pruebas de ensamblaje, con el perno Guía.

La prueba de software consistió en validar los datos de entrada para asegurarse de que en realidad no se dejó ninguna casilla en blanco. En caso contrario la aplicación muestra un mensaje de error y no realizará la prueba. La siguiente verificación consiste en detectar el $\mu \mathrm{C}$ y la tarjeta de prueba, si alguno de estos dispositivos no está presente la prueba no se debe realizar y debe notificarse un mensaje de error. La siguiente revisión consiste verificar que la aplicación añada el registro de la prueba que se realice y que pueda ser abierto con el botón de la interfaz. La prueba demostró que la aplicación crea correctamente el reporte, registra y abre el archivo desde la interfaz. La siguiente prueba consiste en revisar que los indicadores cambien al color correspondiente al resultado de las pruebas. Un indicador en gris significa que la prueba aún no se realiza, uno rojo muestra que no pasó y verde que si pasó. Todo esto se indica en la figura 12. Con las dos pruebas anteriores se determinó el tiempo promedio que tarda el sistema en realizar la validación de una tarjeta, este tiempo fue en promedio de 30s. El tiempo obtenido en las pruebas puede variar si el sistema se ejecuta en una máquina diferente debido al rendimiento de la misma. 

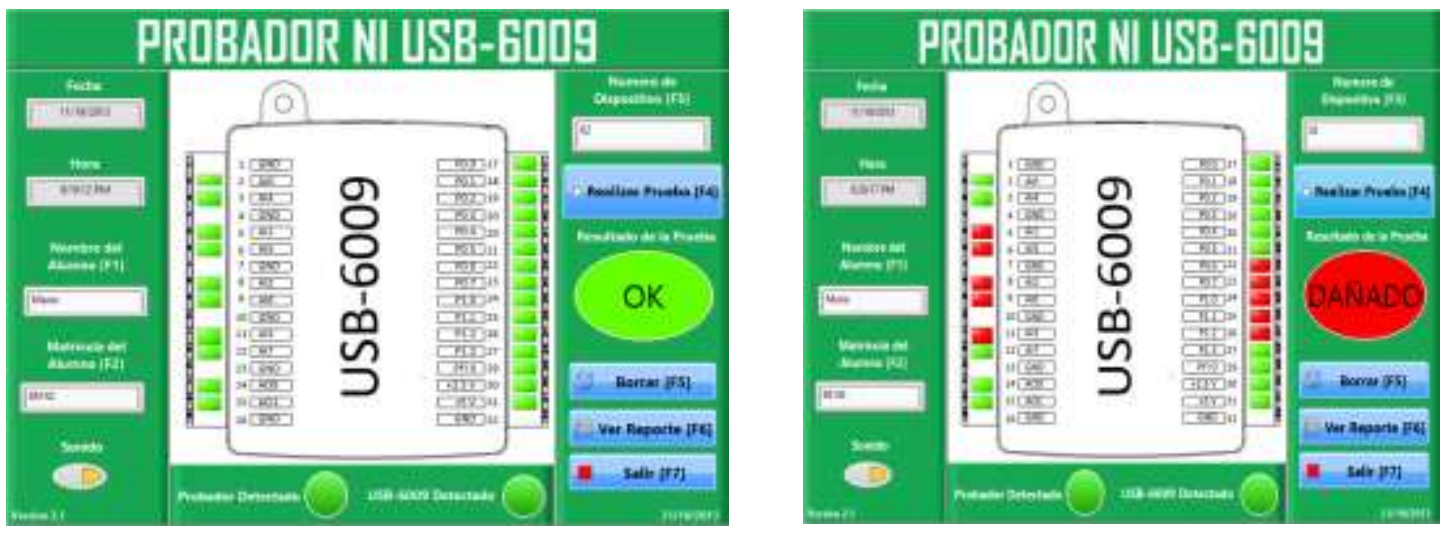

Fig. 12: Prueba a tarjeta en buen estado y mal estado.

\section{CONCLUSIONES}

Del desarrollo de este trabajo se obtienen las siguientes conclusiones: a) Se elaboró un PCB de doble cara lo que redujo el tamaño del circuito y se facilita el remplazo de algunos circuitos integrados al utilizar encapsulado en línea dual (DIP); b) El circuito es alimentado directamente del puerto USB de la PC por lo que no requiere una fuente externa, c) El prototipo es de fácil manejo para el usuario, ya que solo se limita a cambiar la tarjeta de prueba en cada validación y la interfaz es amigable y relativamente fácil de usar, d) El tiempo de respuesta del sistema es suficiente para lograr la prueba en el proceso de préstamo y entrega del producto, e) Este sistema utiliza un $\mu \mathrm{C}$ lo que lo hace económico, y la tarjeta desarrollada se diseñó con software libre y freeware y f) El sistema desarrollado cumplió con el objetivo de realizar la prueba al momento de préstamo y entrega de la misma con lo que Alumnos y Profesores se han visto beneficiados ya que desde su implementación no se han encontrado fallas de tarjetas.

\section{REFERENCIAS}

CadSoft Eagle, (en línea), acceso: 9 de Abril del 2016, Disponible: http://www.cadsoftusa.com/, (2011) Gurrola, O., F. Enríquez, R. Ramírez and R. Lazaro, "LabVIEW Proyecto Final: Probador USB-6009", Depto de Ing. Eléctrica y Computación, Diplomado Program. Gráfica en LabVIEW, Ciudad Juárez, México, (2013)

Hurtado, L.L; Castrillon, O.D y Olivar, G. Una Metodología Automatizada para la Evaluación de Usabilidad de Interfaces de Supervisión Industrial, doi: 10.4067/S0718-07642013000400011, Inf. tecnol., (en línea), 24(4), pp. 95-104, ISSN 0718-0764, (2013)

Issa A. y P. O'Halloran, Construyendo un Sistema de Prueba Automatizado de Manufactura para Amplificación de Audio Utilizando NI LabVIEW y NI TestStand". http://sine.ni.com/cs/app/doc/p/id/cs-12901 (2012)

Jie Y., Pengcheng L., y otros 6 autores. Performance analysis of high-speed parallel optical transmission module based on LabVIEW platform-based test system, doi: 10.1109/ICoOM.2015.7398799, Int. Conference on Optoelectronics and Microelectronics (ICOM), 178-182, Changchun, China, 16 a 18 de Julio (2015)

Jin-yong Yao, Chao Du, Design of fuel valve automatic performance test system based on LabVIEW, doi: 10.1109/IEEM.2015.7385616, Ind. Eng. and Eng. Management (IEEM), 99-103, Singapore (2015)

Liu Jinmei, The testing system of electronic equipment design and implementation based on LabVIEW, doi: 10.1109/CAC.2015.7382877, Chinese Automation Congress, 2247-2250, Wuhan, China, (2015)

National Instruments, Demos de Dispositivo Multifunción USB-6009 DAQ de bajo costo, acceso: 9 de Abril del 2016, Disponible: http://www.ni.com/white-paper/8985/es/, (2013)

National Instruments, User Guide NI, USB-6008/6009, acceso: 9 de Abril del 2016, (En línea), Disponible: http://www.ni.com/pdf/manuals/371303n.pdf, (2005)

Osh Park, (En línea), Disponible: http://oshpark.com/pricing (2013)

Ramirez, A.C; Alique, J.R y Bustillo, A. Dispositivo para la Evaluación y Diagnóstico del Estado del Cabezal en Centros de Mecanizado de Alta Velocidad, doi: 10.4067/S0718-07642006000400005, Inf. Tecno.l., (en línea), 17(4), pp. 23-28, ISSN 0718-0764, (2006)

Silicon Laboratories, Hoja de Especificaciones del CP2102, (en línea), acceso: 9 de Abril del 2016, Disponible: https://www.silabs.com/Support\%20Documents/TechnicalDocs/CP2102-9.pdf, (2013)

Texas Instrument, Hoja de especificaciones del TLV5628, (en línea), acceso: 9 de Abril del 2016, Disponible: http://www.ti.com/lit/ds/symlink/tlc5628.pdf, (1994) 